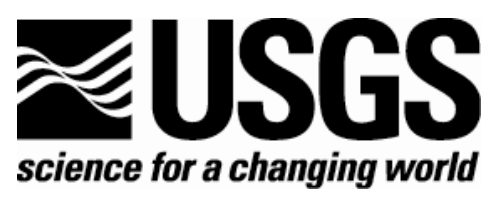

\title{
Bathychronology: Reconstructing Historical Sedimentation from Bathymetric Data in a GIS
}

By Shawn A. Higgins, Bruce E. Jaffe, and Richard E. Smith

Open-File Report 2005-1273 


\section{U.S. Department of the Interior \\ Gale A. Norton, Secretary}

U.S. Geological Survey

P. Patrick Leahy, Acting Director

\section{U.S. Geological Survey, Reston, Virginia 2005}

Suggested citation:

Higgins, S. , Jaffe, B., and Smith, R. (2005) Bathychronology: Reconstructing Historical Sedimentation from Bathymetric Data in a GIS. U.S. Geological Survey Open-File Report 2005-1273, 18p.

[URL: http://pubs.usgs.gov/of/2005/1273]

For product and ordering information:

World Wide Web: http://www.usgs.gov/pubprod

Telephone: 1-888-ASK-USGS

For more information on the USGS - the Federal source for science about the Earth, its natural and living resources, natural hazards, and the environment:

World Wide Web: http://www.usgs.gov

Telephone: 1-888-ASK-USGS

Any use of trade, firm, or product names is for descriptive purposes only and does not imply endorsement by the U.S. Government 


\section{Table of Contents}

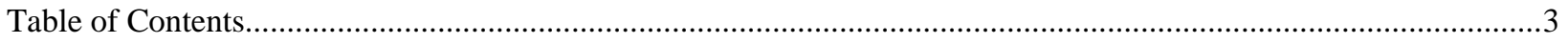

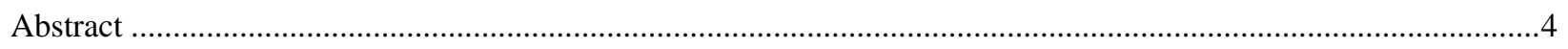

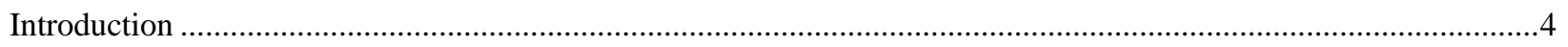

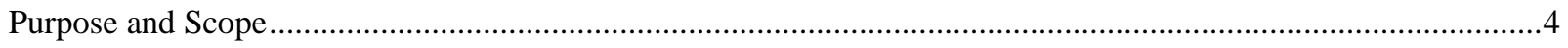

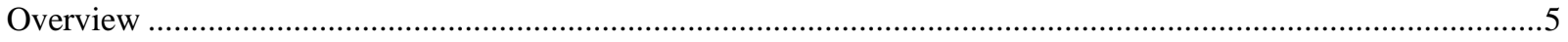

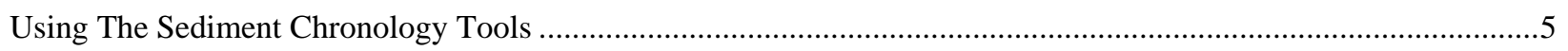

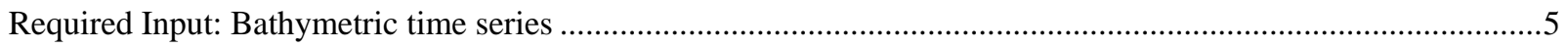

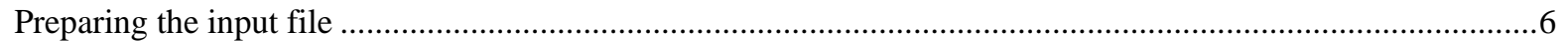

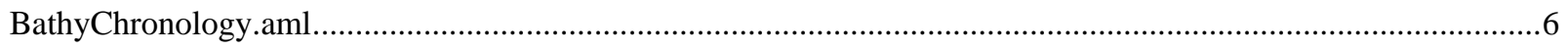

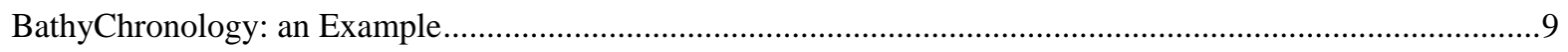

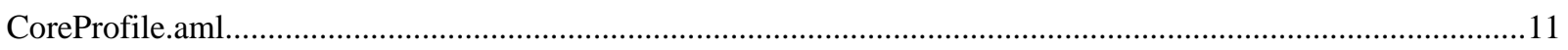

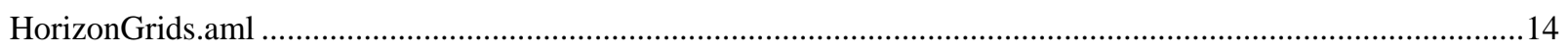

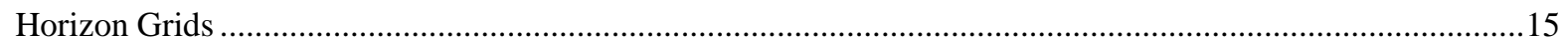

Overlying Coverage Grids .....................................................................................................................15

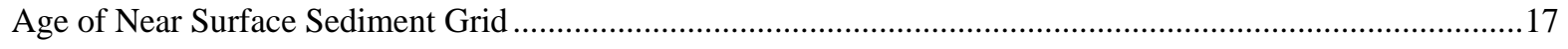

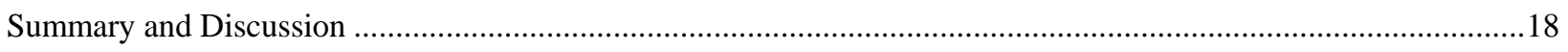

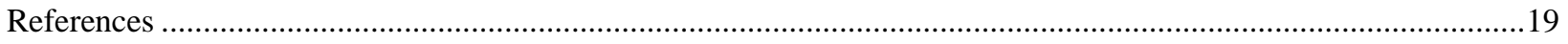




\title{
Bathychronology: Reconstructing Historical Sedimentation from Bathymetric Data in a GIS
}

\author{
By Shawn A. Higgins ${ }^{1}$, Bruce E. Jaffe ${ }^{2}$, and Richard E. Smith ${ }^{3}$ \\ ${ }_{2}^{1}$ Department of Geography, University of Wisconsin-Madison \\ ${ }^{2}$ U.S. Geological Survey Pacific Science Center, Santa Cruz, CA \\ ${ }^{3}$ U.S. Geological Survey Water Resources Division, Menlo Park, CA
}

\begin{abstract}
A collection of computer programs designed to reconstruct a sediment age profile from a time series of bathymetric data in a GIS are presented. The programs utilize an approach called Bathychronology, a technique used to synthesize sediment gains/losses from the time series of bathymetric changes and divide historical deposition into temporal horizons that are separated by survey dates. The application of these programs as tools to reconstruct the profile of a sediment core, map the distribution of horizons from particular time periods, or map the distribution of sediment ages across a surface are discussed. The programs were developed using a historical time series of bathymetric data for San Pablo Bay, California, however, the logic and proper usage of each program are presented for users to apply the programs to other data sets.
\end{abstract}

\section{Introduction}

Sediments delivered to a coastal bay or estuary tend to settle out of suspension and accrete as seafloor deposits. The ultimate fate of these sediments is of interest because 1) sedimentation impacts navigation of coastal waterways, 2) hydrophobic contaminants such as mercury and PCBs adsorb to suspended particles and accumulate in the sedimentary bed, and 3) patterns and rates of sedimentation reflect changes in the environment. The processes controlling sediment transport and deposition are complex and difficult to model. Tools that allow us to reconstruct the distribution and chronology of recent sediments can therefore improve our understanding of the geomorphic processes shaping the seafloor, the potential extent of contamination from environmental pollution, and the likely impacts of environmental changes brought on by natural and anthropogenic forcings.

\section{Purpose and Scope}

The purpose of this report is to describe a technique to reconstruct the depositional history of an area by analyzing the chronology of bathymetric (depth) changes derived from the historical record of hydrographic surveys. Additionally, this report serves to provide documentation about the development and usage of computer programs that automate the reconstruction process. Emphasis is placed on the methodology and application of these programs. 


\section{Overview}

The sediment chronology tools described in this report are computer programs that automate tasks within a geographic information system (GIS). The computer programs are written in the Arc Macro computing Language (AML) and designed to function with the Environmental Systems Research Institute's (ESRI) ArcInfo ${ }^{1}$ Workstation software package. The sediment chronology tools CoreProfile and HorizonGrids both utilize a third program called BathyChronology that analyzes a time-series of bathymetric changes within a GIS and reconstructs a chronology of historical sedimentation based on those changes. The chronology is presented in the form of depositional horizons that stratify the total sedimentation during the historic period into layers. Horizons in this case are defined by the time of deposition and do not reflect sedimentologic characteristics. If the time series of bathymetric changes included surveys in 1925, 1950, 1975, and 2000, for example, the 1950 horizon (denoted hzn1950) represents a layer of sediment deposited between 1950 and 1975.

The CoreProfile program calls on BathyChronology to reconstruct the depositional history at a user specified location(s). CoreProfile then produces a graphic display of the stratigraphic profile in cross-section. HorizonGrids calls on BathyChronology to reconstruct the depositional history for the entire surface of an area being analyzed. HorizonGrids then produces a series of output grids that represent the thickness of individual horizons.

CoreProfile and HorizonGrids are tools that can be used to investigate the distribution and chronology of historical sedimentation. Applications of the sediment chronology tools include: modeling the 3-dimensional form of individual horizons within the sediment bed, mapping the distribution of sediment ages across the surface, and predicting the stratigraphic profile of a hypothetical core sample. The following sections provide more detailed descriptions of the required input data and methodology used in the application of the programs.

\section{Using The Sediment Chronology Tools}

\section{Required Input: Bathymetric time series}

An ArcInfo workspace that contains a time series of bathymetric surface models must be created prior to using the sediment chronology tools. The surface models are spatially referenced grids that store information about the subaqueous terrain of a water body (see Jaffe et al., 1998; Cappiella et al., 1999; Foxgrover, et al. 2004). Grids in the time series correspond to survey dates in the historical record of hydrographic surveys. The grids are composed of square cells arranged in a Cartesian matrix. Each cell is given a value that reflects elevation relative to a vertical datum.

Data from Jaffe et al.’s (1998) analysis of bathymetric change in San Pablo Bay, California was used to develop and test the sediment chronology tools. The time series for this dataset contained six, 50 meter resolution bathymetric grids derived from hydrographic survey data and corrected to a common vertical datum. The sediment chronology tools were designed, however, to be compatible with other datasets of different resolutions. The programs will function with any time series of surface models provided the following conditions are met:

- Series contains a minimum of three grids

- Grids must use the integer data type (no floating point)

- Grids must reference the same coordinate system and units

- Grids must be adjusted to a common vertical datum

- Grid values (bathymetry) should be stored in centimeters

\footnotetext{
${ }^{1}$ The use of trade names is for descriptive purposes only and does not imply endorsement by the U.S. Government.
} 
- Depths below the vertical datum must be negative values

- Elevations above the vertical datum must be positive values

The grids in the bathymetric time series should be saved in a single ArcInfo workspace. This can be the workspace from which the sediment chronology tools are run or a separate workspace on the hard drive.

\section{Preparing the input file}

The sediment chronology tools require a list of input grids and survey years from a file named input.txt in the current workspace. Begin by designating a new or existing workspace as the working directory. Use a text editor to create a new file named input.txt in the workspace. List the bathymetric time series in the input file in ascending order (most recent surface at the bottom). Each line in the file should contain a grid name and the corresponding survey year for the data in that grid (Fig. 1). Format the text using a comma to separate each item (grid name, survey year). Use full pathnames if the grids are stored in a separate workspace.

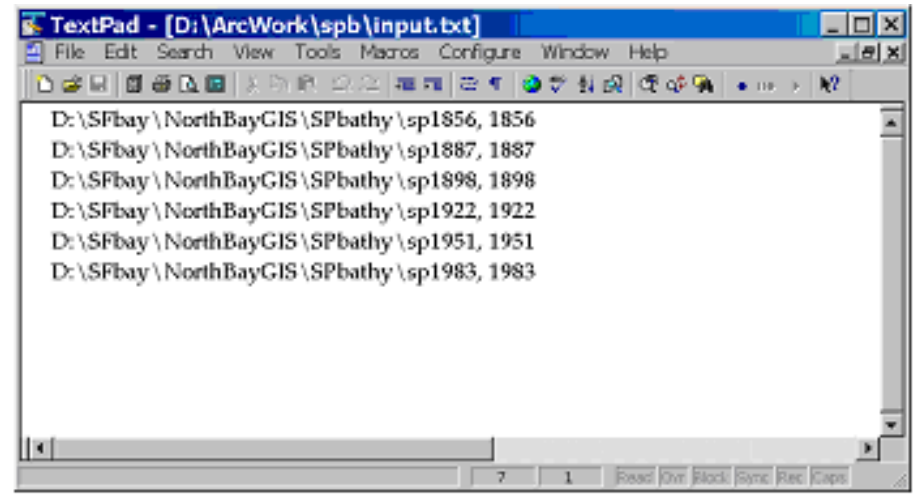

Figure 1. Screenshot of sample input file.

\section{BathyChronology.aml}

Defining depositional horizons from a series of bathymetric surfaces requires that changes in bathymetry over time result from sedimentation or erosion. A decrease in depth between two time periods (resulting in a shallower bay) indicates sediment deposition while an increase in depth (resulting in a deeper bay) indicates sediment erosion. Other factors contributing to bathymetric change such as subsidence, dredging, or vertical datum differences must be accounted for prior to using the methodology presented here.

The reconstruction of depositional horizons occurs in the BathyChronology program. The program takes the series of z-values (bathymetry) for the grid cell containing a specific $\mathrm{x}, \mathrm{y}$ coordinate pair and stratifies any historical sedimentation at that location into depositional horizons. The program's output is a series of horizon files (one file for each depositional horizon) that store the horizon information in the format ' $\mathrm{z}, \mathrm{x}, \mathrm{y}$ ' where $\mathrm{z}$ is the horizon thickness and $\mathrm{x}, \mathrm{y}$ are geographic coordinates. BathyChronology is not designed as a stand- alone program. Instead, the program is nested inside other scripts that read user input and further process the output (see description of CoreProfile.aml and HorizonGrids.aml). Since the BathyChronology program contains the core function of the sediment chronology tools, however, the logic used to reconstruct depositional horizons will be discussed first. 
The process of reconstructing depositional horizons begins by creating a variable array $\mathrm{z} 1, \mathrm{z} 2, \ldots, \mathrm{zn}$ (where $\mathrm{n}$ is the number of grids in the time series) to store the change in bathymetry over time at the specified $x$, y location. The values in the array are obtained using the CELLVALUE command for each grid in the time series. The next step is to identify the deepest z-value in the array. If the deepest value corresponds to the most recent grid in the time series, then erosion has lowered the surface below all other historical surfaces and there will be no historical sedimentation. If, on the other hand, the deepest z-value occurs earlier in the time series, then historical sediment has accreted at that location.

After identifying the deepest z-value in the array, an aml variable called Base is initialized to the position in the time series corresponding to the deepest $z$-value (i.e. if $z 1$ is deepest, then Base = 1, if $z 2$ is deepest, then Base = 2, etc.). A looping sequence (Fig. 2) is then begun to reconstruct the depositional horizon corresponding to the Base year in the time series. Inside of the loop, two new aml variables, zBase and zNext, are created. zBase is always set to equal the z-value of the Base year. zNext is set to the deepest $\mathrm{z}$-value among all grids following the Base year in the time series. Using this method allows the horizon thickness to account for subsequent erosional periods that truncate the initial deposition. The thickness of the horizon is calculated by differencing zBase from zNext. The result is written, along with the $\mathrm{x}, \mathrm{y}$ coordinates of the location, to the output horizon file (if Base $=1$, then the output is written to 'hzn1', if Base = 2, then the output is written to 'hzn2', etc.).

At the bottom of the loop (Fig. 2), the variable Base is reset to equal the position in the time series corresponding to zNext (i.e. if $z N e x t=\mathrm{z} 4$, then Base $=4$ ). A condition is then tested before the loop repeats. If Base is equal to the last position in the time series $(n)$, then there is no additional sedimentation and the loop stops. If Base $<n$, then the loop repeats. A new zBase and zNext are set and a new horizon is written to another horizon file. The loop continues until Base $=n$. 


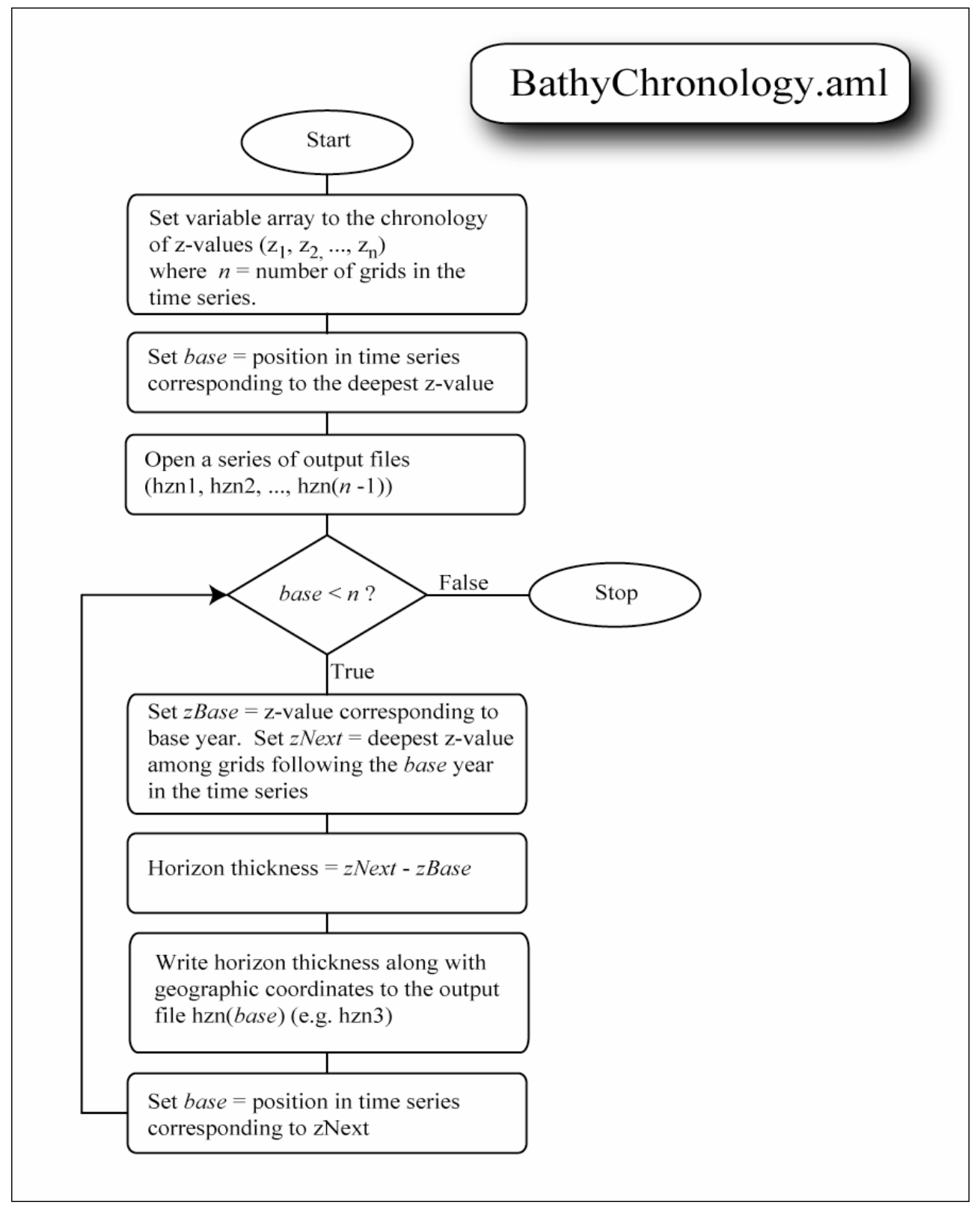

Figure 2. Flowchart diagram of the logic and procedures in the BathyChronology computer program. 


\section{BathyChronology: an Example}

The following example illustrates the reconstruction process for a sample $\mathrm{x}, \mathrm{y}$ location. Two horizons are reconstructed from the historical sedimentation (Fig. 3).

- The array of z-values is created for the sample location

- $\mathrm{Z} 1=-300$

$Z 2=-200$

$\mathrm{Z3}=-180$

$Z 4=-210$

$\mathrm{Z} 5=-220$

$Z 6=-190$

- Base is initialized to the position of the deepest z-value

- $\quad$ Base $=1$

- Zbase is set to the z-value of the Base year

- Zbase $=-300$

- ZNext is set to the deepest $\mathrm{z}$-value among grids following the Base year

- $\quad$ ZNext $=-220$

- The depositional horizon corresponding to the Base year is calculated

- Hzn1 = zNext - zBase

- Hzn1 = -300 - -220

- $\operatorname{Hzn} 1=80 \mathrm{~cm}$

- The horizon thickness is written to the horizon file (hzn1)

- Base is reset to the position corresponding to zNext

- $\quad$ Base $=5$

- $\quad$ The condition at the top of the loop is tested (Base $<n$ )

- Since Base is not the last grid in the series, the loop repeats

- $\quad$ Zbase is set to the $\mathrm{z}$-value of the Base year

- Zbase $=-220$

- ZNext is set to the deepest z-value among grids following the Base year

- ZNext $=-190$

- The depositional horizon corresponding to the Base year is calculated

- Hzn5 = zNext - zBase

- $\mathrm{Hzn} 5=-220--190$

- $\mathrm{Hzn} 5=30 \mathrm{~cm}$

- The horizon thickness is written to the horizon file (hzn5)

- Base is reset to the position corresponding to zNext

- $\quad$ Base $=6$

- The condition at the top of the loop is tested (Base $<n)$

- $\quad$ Since $n=6$, the loop stops 


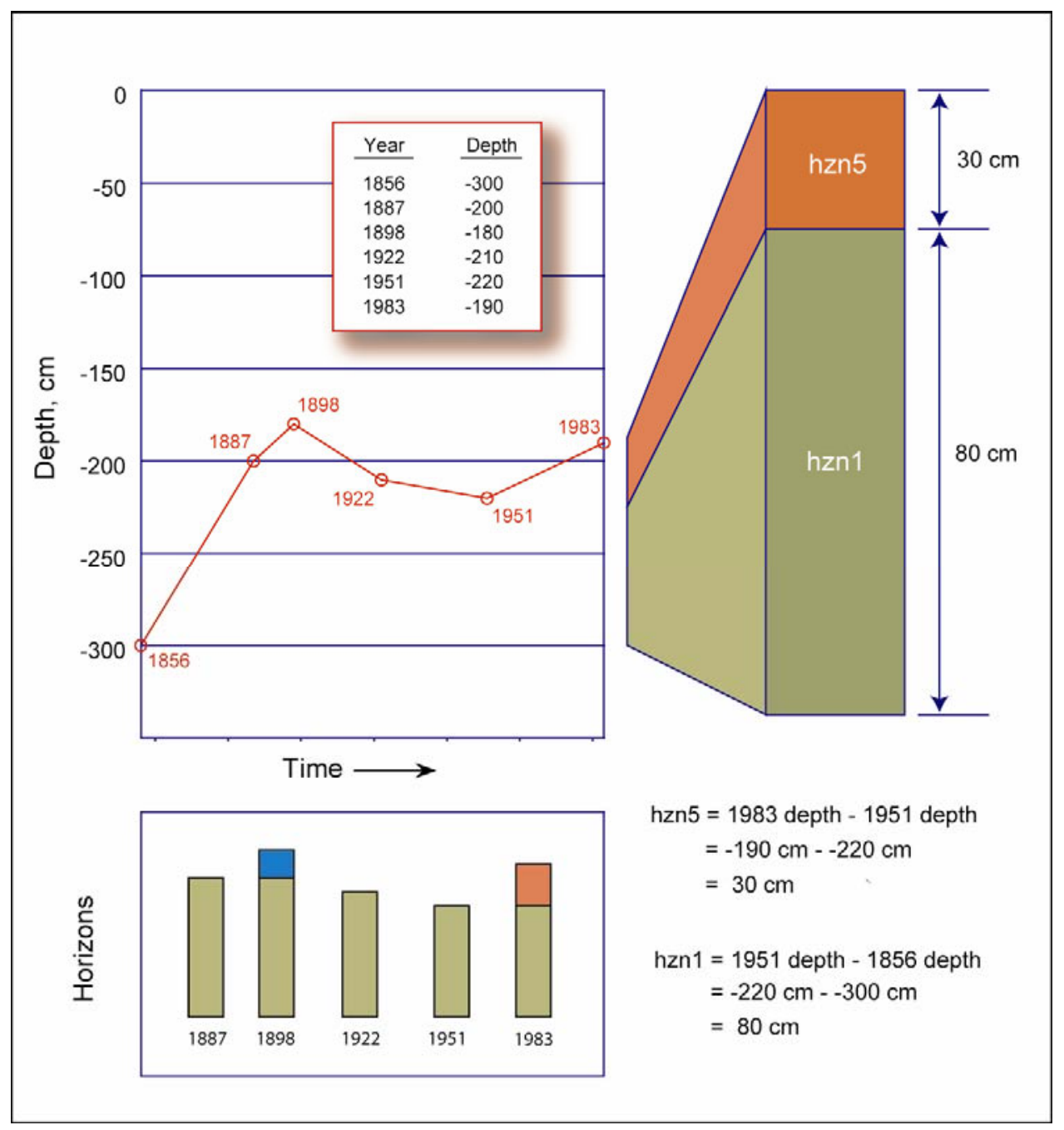

Figure 3. Example of reconstructed depositional horizons for a sample bathymetric time series. 


\section{CoreProfile.aml}

The CoreProfile program reconstructs the depositional history at a user specified location and produces a graphic display of the stratigraphic profile in cross-section (Fig. 4). The program is executed using the \&RUN directive from the command line prompt in ArcInfo. Processing time for CoreProfile is relatively fast. Each location is typically processed in less than 30 seconds.

CoreProfile begins by reading the contents of the input file and setting the grid names and survey years to an array of AML variables. Next, the program identifies the geographic coordinates of the location(s) to analyze. The user is asked at the command prompt if the coordinates will be entered from a file, through the keyboard, or with the mouse. If the user responds 'file' at the prompt, then the program will ask for the name of a text file saved in the working directory that contains a list of coordinate values formatted as $I D, X, Y$. The ID is a user-defined name to identify the location and $\mathrm{X}, \mathrm{Y}$ are the geographic coordinates. The program then reads the text file and saves the data to AML variables. If the user responds 'keyboard' to the initial prompt, then the program will ask that the geographic coordinates be entered interactively to a series of prompts at the command line. If the user responds 'mouse' to the initial prompt regarding coordinate input, then the program executes a routine that draws a graphic of the most recent grid to the screen and captures coordinates in response to user clicks with the mouse.

After retrieving information about the bathymetry grids and geographic locations to analyze, CoreProfile proceeds to reconstruct depostitional horizons by passing information to the BathyChronology program. The horizon files output from BathyChronology are then read by CoreProfile. A routine is then executed to create a graphical representation of the predicted stratigraphy using tools available in the ArcPlot module. The graphic is plotted to the screen for immediate viewing and is also saved as an Encapsulated PostScript (.eps) file for editing and printing. The graphic contains three elements: a cross-sectional profile, a table listing the chronology of bathymetric changes, and a map marking the geographic location of the reconstruction (Fig 5).

The cross-sectional profile has one vertical axis on the left indicating depth in the core and a second axis on the right indicating the year of deposition for sediments at the top and bottom of each horizon. The year at the bottom of each horizon is always the same as the survey year from which deposition was measured. If all of the initial deposition from that period remains, then the year at the top of the horizon is equal to the next consecutive survey year in the time-series. If, however, a portion of the initial deposition from that period has been removed by a later erosional period, then the year at the top of the horizon is determined by linear interpolation of the remaining deposition. That is, if $50 \%$ of the initial deposition remains and the period of initial deposition was 30 years, then $50 \%$ of 30 years (15 years) is added to the survey year from which deposition began.

The horizon thickness (in centimeters) is shown on the cross-sectional profile. Additionally, a +/- value is included to evaluate the spatial homogeneity of each reconstructed horizon. The +/- value represents the standard deviation of initial deposition for a $3 \times 3$ grid cell neighborhood around the location of the reconstruction. A high standard deviation relative to the horizon thickness would indicate an area of high spatial variation such as the margin of a channel or steep slope. Such a value should flag the user to be cautious as a small change in location could produce a large change in the estimated profile. 


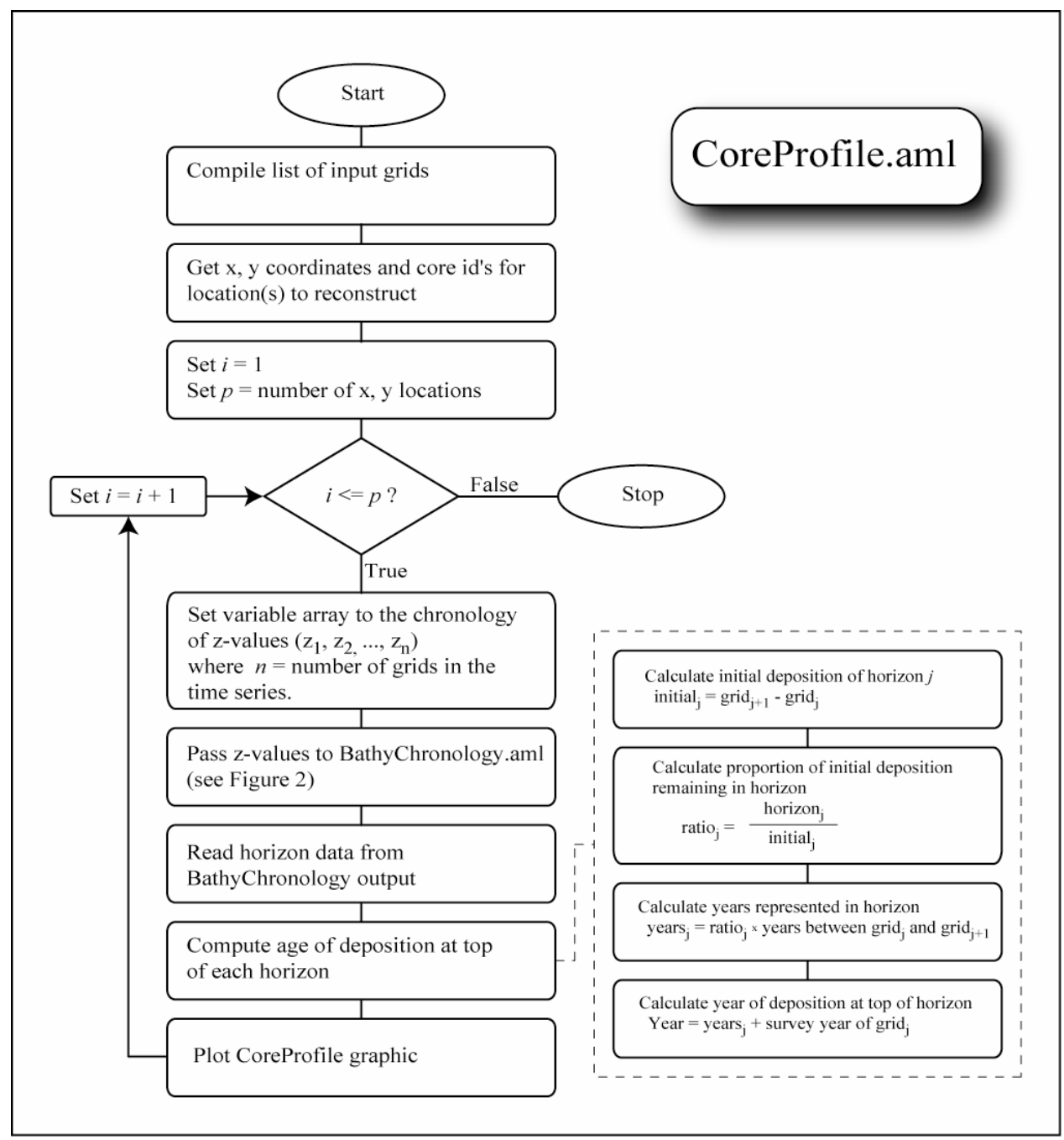

Figure 4. Flowchart diagram of the logic and procedures in the CoreProfile computer program. 


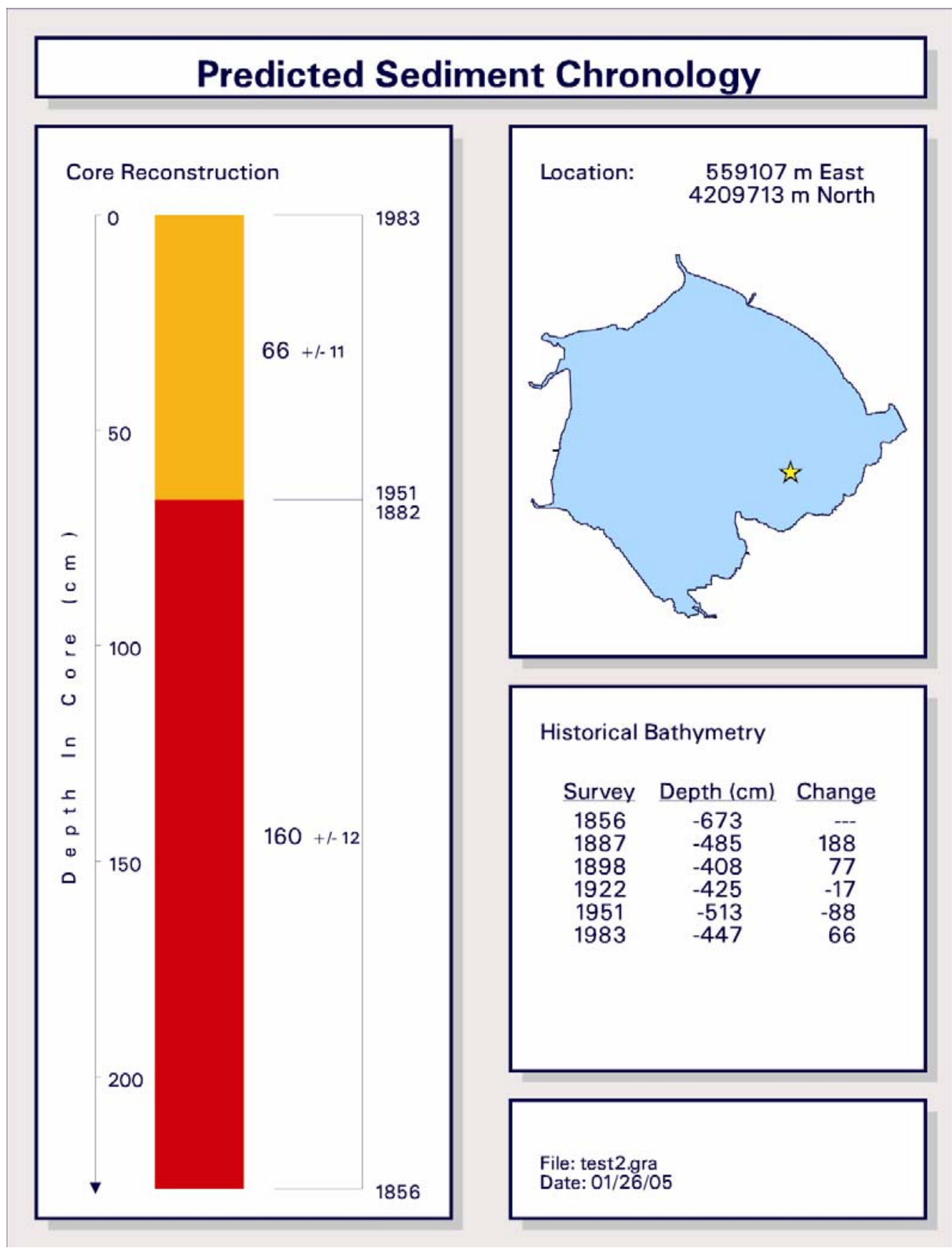

Figure 5. Sample output graphic from the CoreProfile computer program. 


\section{HorizonGrids.aml}

The HorizonGrids program reconstructs a depositional history for the entire surface of an area being analyzed (Fig. 6). The program produces three outputs: 1) a series of grids representing the individual depositional horizons, 2) a series of grids representing the coverage of more recent sediment overlying each horizon in the stratigraphic profile, and 3) a grid to represent the spatial distribution of sediment ages near the bathymetric surface. Similar to CoreProfile, the HorizonGrids program is executed using the \&RUN directive from the command line prompt in ArcInfo. Because the program repeats once for every cell in the grid surface, HorizonGrids can take a long time to run. Test runs using a $1.6 \mathrm{GHz}$ Pentium 4 processor on a 50-meter grid covering approximately $700 \mathrm{~km}^{2}$ (540 rows x 540 columns) lasted nearly 10 hours.

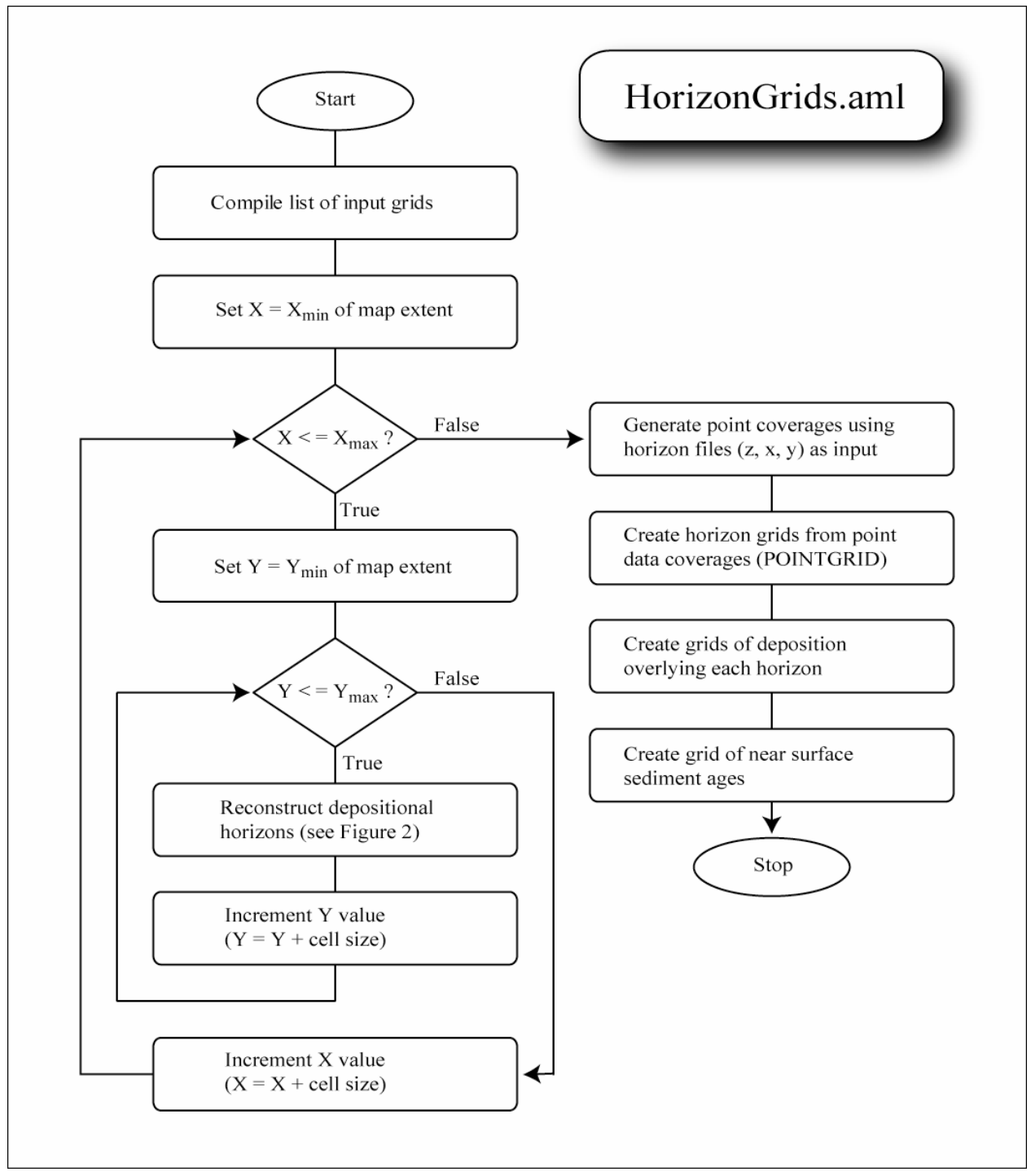

Figure 6. Flowchart diagram of the logic and procedures in the HorizonGrids computer program. 


\section{Horizon Grids}

In order to map the spatial distribution of sediments from different historic periods, the HorizonGrids program creates a series of grids that represent horizon thickness. HorizonGrids begins by reading the contents of the input file and setting the grid names and survey years to an array of AML variables. A nested loop is then executed to process each cell in the grid surface individually (Fig. 6). To begin, aml variables $X$ and $Y$ are initialized to the minimum $\mathrm{X}$ coordinate and minimum $\mathrm{Y}$-coordinate of the spatial extent covered by the input grids. The BathyChronology program is then called on to reconstruct depositional horizons at the location specified by $X$ and $Y$. Depositional horizons, along with the $\mathrm{x}, \mathrm{y}$ coordinate pair, are written to the corresponding horizon files. As the loop repeats, $X$ and $Y$ values are incremented by the cellsize of the grid until every cell has been processed.

A series of horizon files are created by the iterations of BathyChronology. An individual horizon file can contain many lines of text, each line listing the horizon thickness at a particular $\mathrm{x}$, y location. After the looping sequence that processes each grid cell, the data in the horizon files are used as input to generate point coverages. For a particular horizon, one point feature will be created for every grid cell for which deposition exists. The POINTGRID command then creates a gridded surface from each point coverage (Fig. 7). The grid values will be equal to the horizon thicknesses. The horizon grids follow the naming convention 'hzn1951', where 1951 is the survey year from which deposition is reconstructed (Base in BathyChronology).

\section{Overlying Coverage Grids}

In addition to knowing the thickness of a depositional horizon at a particular location, it is useful to know how close that horizon is to the modern surface. If one is concerned with sediments from a specific time period, for example, are those sediments deeply buried beneath more recent sediments or are they near the surface? The grids of overlying sediment coverage (Fig. 7) provide this information.

The process of creating the grids is simply a matter of summing the depositional horizon grids that follow the horizon sequentially. If there are five depositional horizon grids in the series: hzn1856, hzn1887, hzn1898, hzn1922, hzn1951, and hzn1983, then the sediment coverage grid for the first depositional horizon is calculated as:

$$
\text { hzn1856cvg = hzn1887 + hzn1898 + hzn1922 + hzn1951 + hzn1983 }
$$

and the sediment coverage grid for the next depositional horizon is calculated as:

$$
\text { hzn1887cvg = hzn1898 + hzn1922 + hzn1951 + hzn1983. }
$$

The overlying coverage grids follow the same naming convention used for the horizon grids but add the cvg suffix (e.g. hzn1951cvg). 
Horizon Thickness (m)

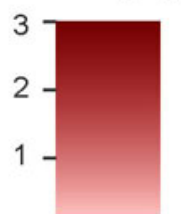

No Deposition No Data
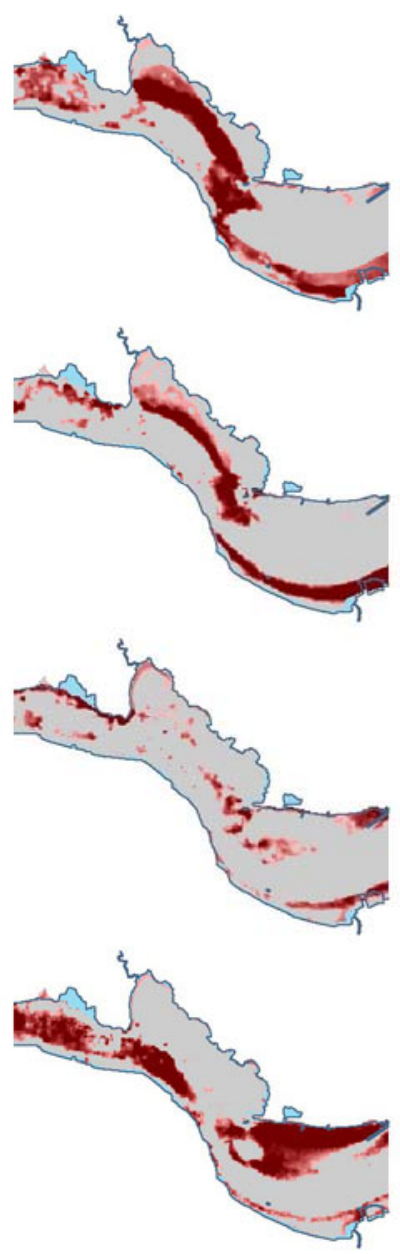

$1 \mathrm{Km}$
Overlying Sediment (m)

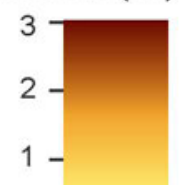

No Deposition No Data

1867-1898

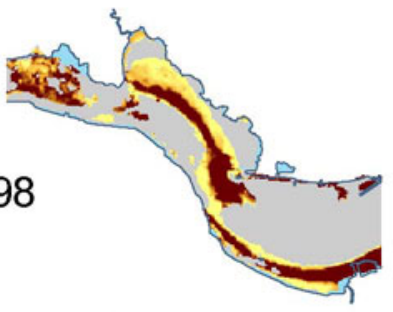

$1898-1922$

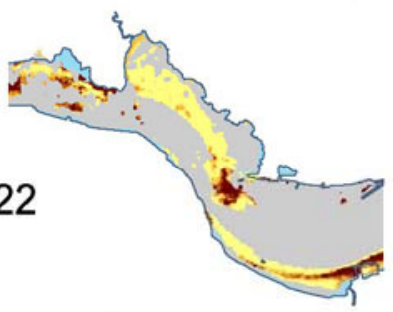

1922-1942

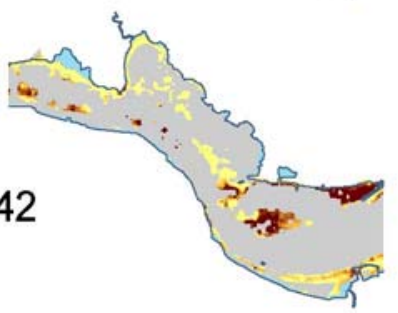

$1942-1990$

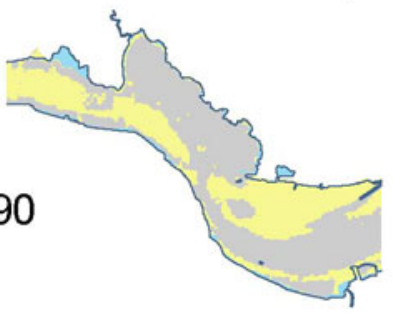

Figure 7. Maps illustrating a sample series of horizon grids along with the corresponding series of overlying coverage grids generated from the HorizonGrids computer program. 


\section{Age of Near Surface Sediment Grid}

The conclusion of the HorizonGrids program creates a grid to model the distribution of sediment ages (referring to year of deposition) across the surface of the study area. This new grid, named 'surfaceage', contains values representing the year of deposition for sediments at the top of the uppermost horizon in the sedimentary profile (Fig. 8). If a given grid cell was depositional during the interval between the two most recent surveys, then the value of that cell on the surfaceage grid will be equal to the year of the most recent survey. If, however, a grid cell was erosional during the most recent period of record, then it is expected that sediments deposited from an earlier period would be found at the top of the sedimentary profile. In such a case the value is determined by first identifying the uppermost depositional horizon and comparing the horizon thickness to the amount of initial deposition from that period. In calculating the surfaceage value, the program assumes a constant rate of sedimentation between the two surveys defining that period. The number of years spanned during that period is then multiplied by the proportion of remaining sediments and added to the beginning year of sedimentation to estimate the year of deposition for the top of the horizon.

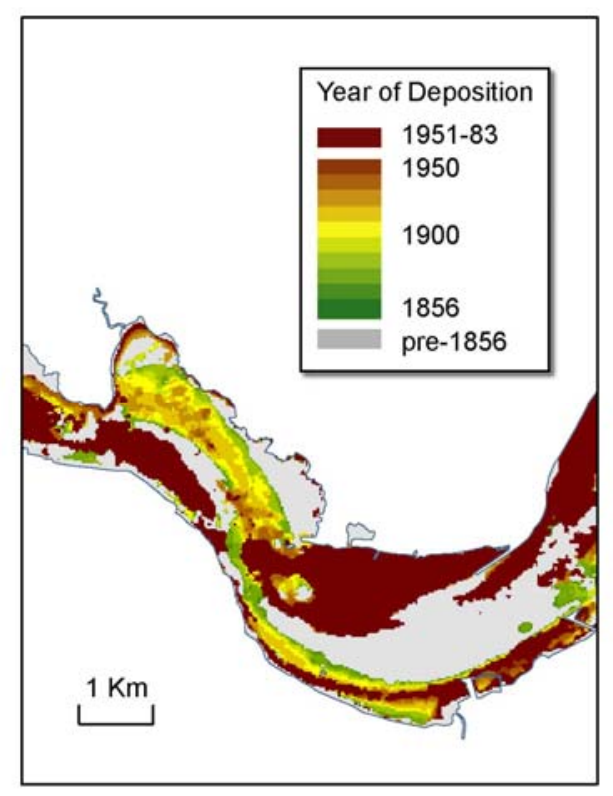

Figure 8. Map illustrating a sample surfaceage grid generated from the horizongrids computer program. 


\section{Summary and Discussion}

The above description of the BathyChronology, CoreProfile and HorizonGrids computer programs presents a new approach to reconstructing a chronology of sedimentation by incorporating historical bathymetry data into a GIS application. Automating the methodology with AML programs has produced powerful tools that can be used to model the distribution of recent sediments. The design intent of the programs focused on making the analysis easily repeatable and adaptable for other regions. It is the aim of this report then to explain the application and correct usage of the sediment chronology tools in such a way that anyone with a dataset similar to the bathymetric time-series described above can use the BathyChronology, CoreProfile, and HorizonGrids programs to analyze the depositional history of an area.

The nature of the input data used in to develop the sediment chronology tools (hydrographic surveys) limited the usefulness of this approach to investigating sedimentation trends that are large in magnitude and detectable with a sampling interval that spans decades. Seasonal and annual variations can obviously not be modeled using data that spans decades. The assumption used in the methodology that sedimentation is constant between surveys is unrealistic but necessary given a lack of data for the intervening period. It is acknowledged that assigning a specific year of deposition for sediments at the top of the horizons and on the surfaceage grid exaggerates the precision of the tools, however, the addition of these data aids in the interpretation of the reconstructed sediment chronologies. When we gain more knowledge regarding the processes controlling interannual variability it will be possible to develop nonlinear methods of interpolation.

It should also be noted that the assumptions used in the computer programs to reconstruct depositional horizons from bathymetric changes simplify the processes affecting the sedimentary profile. There certainly are other factors to consider, such as sediment compaction and biological mixing, when interpreting sediment chronologies. In developing the sediment chronology tools, however, these influences are omitted.

By simplifying the environmental processes affecting deposition in coastal waterways, the sediment chronology programs CoreProfile and HorizonGrids provide insight into the distribution of sediments from the historical period. The results are intended to supplement, not replace, other methods of investigating sediment chronologies. The reconstructions produced by these programs offer a new way to explore the sedimentary deposits hidden beneath the waves. 


\section{Acknowledgements}

This research was supported by the USGS San Francisco Bay Priority Ecosystem Project, Dr. Janet Thompson Project Chief, and the USGS Geologic Division Toxics Program, Dr. James McNeal, Program Coordinator.

\section{References}

Cappiella, K., Malzone, C., Smith, R., and Jaffe, B. (1999) Sedimentation and Bathymetry Changes in Suisun Bay: 1867-1900. U.S. Geological Survey Open-File Report 99-563.

[URL: http://geopubs.wr.usgs.gov/open-file/of99-563]

Foxgrover, A., Higgins, S., Ingraca, M., Jaffe, B., and Smith, R. (2004) Sedimentation, Erosion, and Bathymetric Change in South San Francisco Bay: 1858-1983. U.S. Geological Survey Open-File Report 2004-1192. [URL: http://pubs.usgs.gov/of/2004/1192/]

Jaffe. B., Smith, R., Torresan, L. (1998) Sedimentation and Bathymetric Change in San Pablo Bay: 1856-1983. U.S. Geological Survey Open File Report 98-759.

[URL: http://geopubs.wr.usgs.gov/open-file/of98-759] 